\title{
OSTEOCHONDROMATOSIS OF THE HIP JOINT
}

\author{
Report of a Case \\ GaWad Hamada, Alexandria, Egypt \\ From the Section of Orthopaedics, Farouk I Unitersity, Alcxandria
}

This report is submitted because the patient concerned illustrates rather unusual stages in the development of osteochondromatosis of the hip joint, itself an uncommon condition.

A railway worker, twenty years old, was struck heavily in the right buttock on December 25, 1945. About a month later he began to complain of continuous and increasing pain in the right hip. He attended hospital eight months after the injury. On examination there was limitation of hip movements, which were painful, and radiographs showed a defect in the lower part of the head of the femur with a rather faint shadow resembling that of a loose body in the region of the upper part of the base of the femoral neck (Fig. 1). The hip

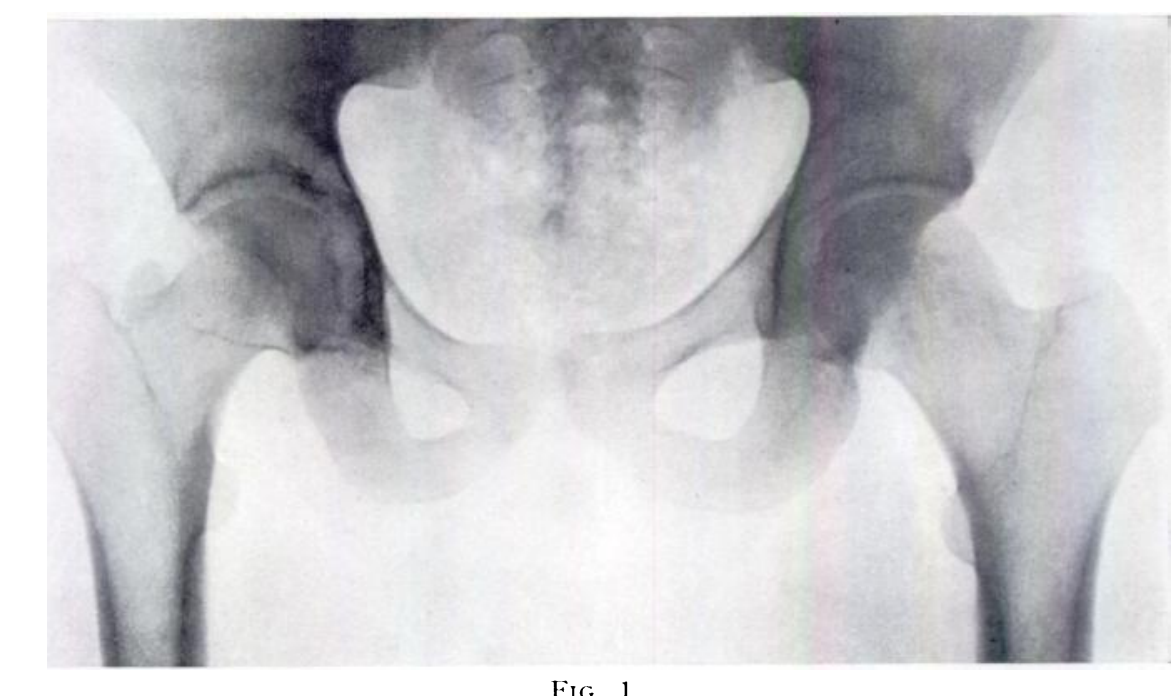

Radiographs of the hip joints seven months after the onset of symptoms. Note, in the right hip, the defect in the lower part of the head of the femur, and the faint shadows at the lower margin of the joint and near the upper border of the femoral neck.

was immobilised in plaster-of-Paris for four months. A second radiograph taken after removal of the plaster showed the loose body rather more clearly outlined in a hip showing disuse atrophy (Fig. 2), but a third film three months later showed massive deposition of opaque "loose" bodies to a quite unexpected degree (Fig. 3).

The hip was explored fifteen months after the onset of symptoms. The " tumour " mass was adherent to the neck of the femur and to the capsule, and had to be gouged away. Complete removal was impossible. The naked-eye appearances are illustrated in Figure 4. The pathological appearances were those of a chondroma.

The patient was last seen three years after the operation. Radiographs (Fig. 5) then showed persisting radio-opaque bodies in the hip, but no massive recurrence and no marked erosion of bone.

vol. 33 B, No. 1, FEBRUARY 1951 


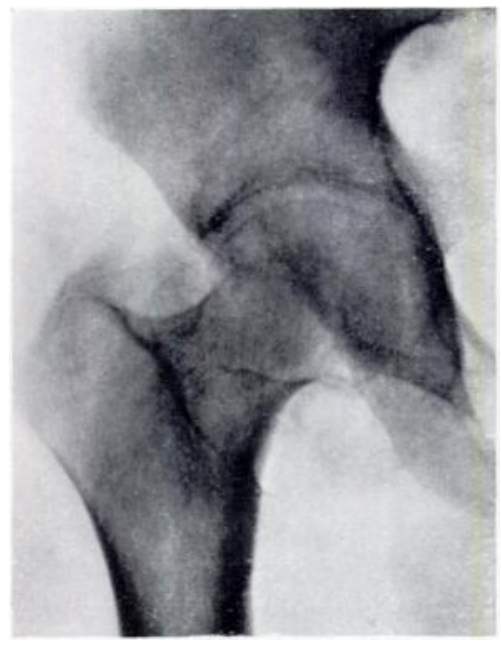

FIG. 2

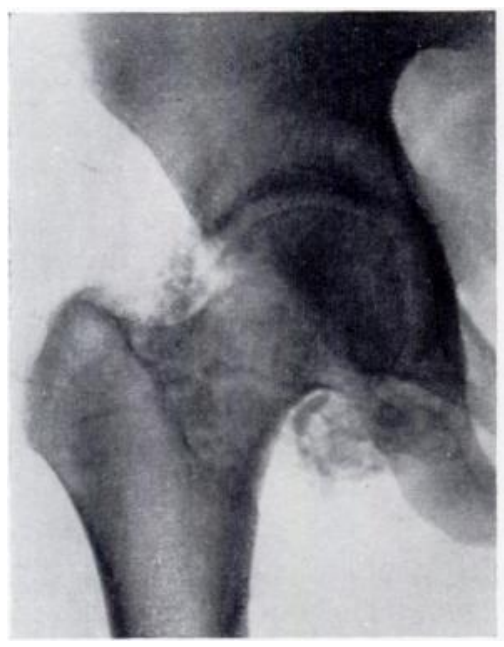

FIG. 3

Figure 2-Four months later. There is faint opaque mottling throughout the area of the hip joint and capsule. Figure 3-Three months later. There is striking increase in calcification of the intracapsular mass.

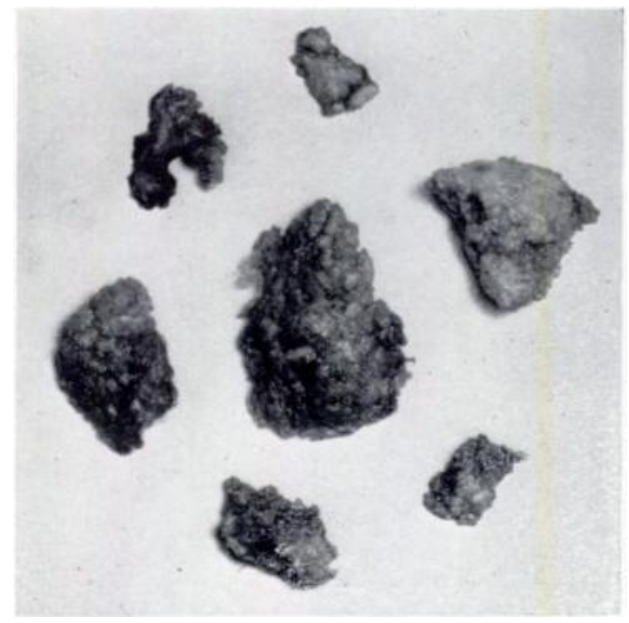

FIG. 4

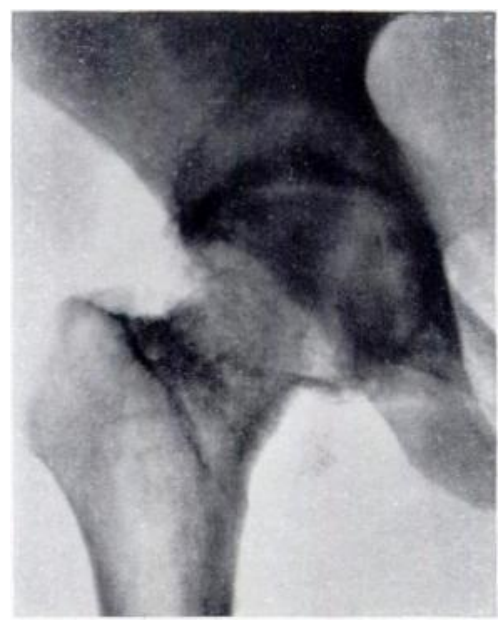

FIG. 5

Figure 4-Photograph of the osteochondromatous mass removed at operation. Figure 5 Radiograph three years after operation. Irregular opacities are still present but there has been no massive recurrence and no further resorption of bone.

\section{COMMENT}

The remarkable change in the radiographic appearances in the three-month interval between the second and third radiographs (Figs. 2 and 3) must be taken to indicate rapid extension of the disease. It was, in fact, so rapid that suspicions of malignancy were raised at the time. But the subsequent history and the result three years later support the suggestion that the condition was simply an osteochondromatosis, with an unusual "explosive" phase in its development. 